\title{
Sponsoring Institution Interests, Not National Plans, Shape Physician Workforce in the United States
}

Diane R. Rittenhouse, MD, MPH; Alexandra S. Ament; Kevin Grumbach, MD

\begin{abstract}
BACKGROUND AND OBJECTIVES: Graduate medical education (GME) determines the composition and distribution of the physician workforce in the United States. Federal and state governments heavily subsidize GME but in most cases do not tie subsidies to national or state physician workforce goals. As a result, GME sponsoring institutions (eg, teaching hospitals, schools of medicine, federally qualified health centers) decide how many and what type of physicians to train. The objective of this study was to better understand the factors that influence decision-making by sponsoring institutions.
\end{abstract}

\begin{abstract}
METHODS: Between May and December 2018, we interviewed 35 national or state GME policy leaders and an additional 26 GME leaders from a purposive sample of four sponsoring institutions. We analyzed interviews following a conventional content analysis approach to identify emergent themes.

RESULTS: When considering investing in GME, we found that sponsoring institutions do not consider national or statewide workforce recommendations. Instead, they weigh multiple factors of concern to their institution, including public GME subsidies, market competition, potential clinical revenues, academic stature, local workforce demands, as well as their own organization's mission/ culture, staffing, financial reserves, educational leadership, teaching resources, and size.
\end{abstract}

CONCLUSIONS: Unless and until the incentives for sponsoring institutions are strongly aligned with national and state physician workforce priorities based on public need, progress on creating a more balanced physician workforce will not occur.

(Fam Med. 2020;52(8):551-6.)

doi: 10.22454/FamMed.2020.507727

Published Online First July 16, 2020

raduate medical education (GME) determines the number, specialty, and geographic distribution of physicians in the United States. GME is heavily subsidized by public funding, with an estimated $\$ 16$ billion coming from federal sources in $2015 .{ }^{1}$ Despite recommendations from several commissions and organizations over the past several decades ${ }^{2-6}$ the largest federal funding source, the Centers for Medicare and Medicaid Services (CMS), has never adopted an explicit national physician workforce plan to guide its funding of GME. ${ }^{\text {? }}$ With no clear link between federal physician workforce policy and federal funding of GME, decisions regarding which physicians to train and where to train them are left to individual sponsoring institutions. ${ }^{8 *}$ This devolution of planning to sponsoring institutions has contributed to a physician workforce in the United States that is concentrated in urban areas and skewed toward specialists at the expense of an adequate number of primary care physicians and psychiatrists. ${ }^{9}$ Yet no studies have systematically investigated the perspectives of decision-makers at sponsoring institutions about the factors that influence their decisions around GME. To better understand those factors, we conducted a qualitative study that involved key informant interviews with GME policy experts and GME leaders at sponsoring institutions.

\section{Methods}

Study Design and Participants

Between May and December 2018, we interviewed 35 national GME policy experts or state GME policy leaders and an additional 26 leaders from a purposive sample of four sponsoring institutions. The four sponsoring institutions were based in California, but were chosen to be representative of the diverse types of sponsoring institutions that exist across the United States. They

From Mathematica, Oakland, CA (Dr Rittenhouse); and the Department of Family and Community Medicine, University of California, San Francisco (Dr Rittenhouse, Dr Grumbach, and Ms Ament). 
included a large university-based urban academic health center, a large community-based integrated delivery system, a small for-profit community hospital consortium, and a small rural community clinic-based teaching health center. Informants interviewed had an in-depth understanding of institutional decisionmaking around GME and included leaders with titles such as GME designated institutional officer (DIO), residency program director, and hospital CEO. Consistent with federal guidelines, University of California, San Francisco does not require institutional review board review for research on organizations involving gathering of information from organizational officials.

\section{Data Collection}

For each interview, we used a uniform semistructured interview and discussion guide tailored to the individual and/or organization, covering topics such as the institution's mission, sources of GME funding, GME affiliations, challenges and benefits of serving as a GME sponsoring institution, and decision-making around expanding or contracting GME programs. We conducted most interviews in person, and some by telephone. Most interviews were recorded and transcribed to supplement detailed interviewer notes. Interviews lasted 45-60 minutes.

\section{Data Analysis}

We analyzed interviews following a conventional content analysis approach to identify emergent themes. Early transcripts were coded independently without the use of qualitative software. Subsequently, authors D.R. and A.A. met regularly to reach consensus on topics, identify discrepancies, refine concepts, and develop emerging themes. Quotes provided exemplify these themes.

\section{Results}

Eleven themes emerged as factors that influence decision making by individual sponsoring institutions (Figure 1). We detail each of these factors in the following sections, and provide exemplar quotes for each.

\section{Public GME Subsidies}

Medicare GME payments account for the vast majority of public spending on GME and provide an important source of revenue for teaching hospitals. Payments to teaching hospitals are not based on the actual costs of administering GME programs, but instead on formulae developed in the 1980s that result in relatively high per-resident payments, creating a strong incentive to remain engaged in GME. However, Medicare capped the number of residents eligible for reimbursement, disincentivizing program growth. Hospitals can spend Medicare GME subsidies in any way they choose because CMS does not require that they spend the monies on GME. In addition, hospitals may use the funds to train residents and fellows in any specialty they choose.

All dollars are green. So the health system gets that $[\mathrm{X}]$ million or whatever it is, and it goes into the

Figure 1: Decision-Making by GME-Sponsoring Institutions Focuses on Many Factors Other Than National and Regional Workforce Needs

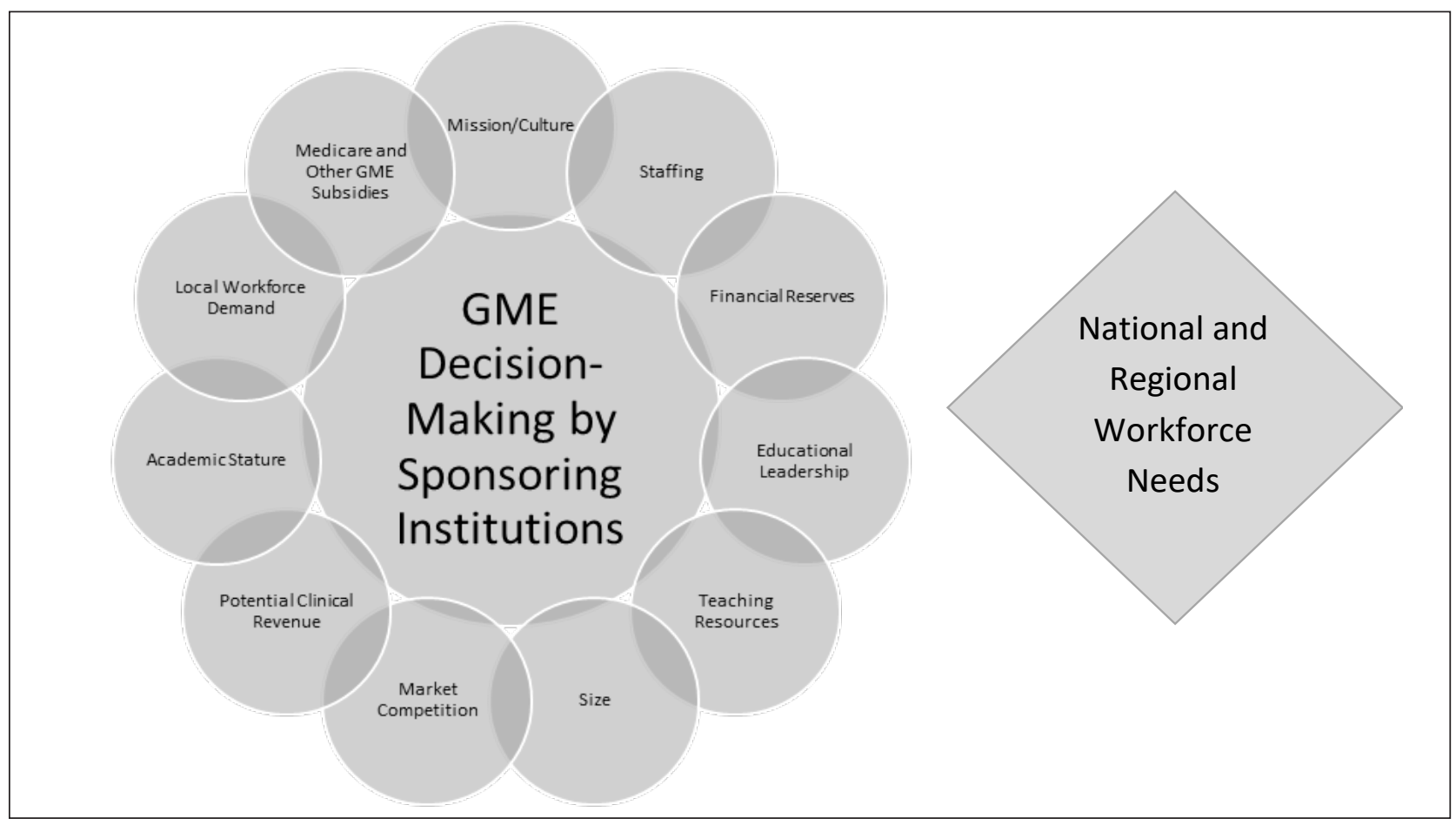


revenue bucket and it's not any different to them... It's just another part of their revenue flow. - Designated Institutional Official \#1

Medicaid is a federal-state program that provides the second largest financial contribution to GME in teaching hospitals. Lack of federal guidance for Medicaid GME funding allows policies to differ in every state. Some states pay for hospitalbased GME in a system of payments similar to Medicare; other states are introducing innovations to increase accountability and transparency. The federal Health Resources and Services Administration's (HRSA's) Teaching Health Center (THC) and Children's Hospital programs are relatively small sources of GME funding, but for some sponsoring institutions (eg, FQHCs and children's hospitals) these may be the only federal GME subsidies for which they are eligible. HRSA funding is awarded to hospitals in the form of grants that are time-limited-an unstable source of funding that leaves GME programs at risk of contracting or closing when grants shrink or disappear.

The fact is we're going to have to go up or down [in number of residents] based on that extra funding because we're not assured it's going to come, and I can't afford to fund a family medicine residency out of operations. -Administrator \#1, Teaching Hospital

Compared to Medicare GME payments, the THC program and some state government GME funding programs are more proscriptive in funding trainees in specialties deemed to be in shortage, such as primary care and psychiatry, and prioritizing funding of residencies preparing trainees for practice in underserved communities. The US Department of Veterans Affairs (VA) invests substantially in GME across the country, typically in partnership with non-VA sponsoring institutions. The VA has targeted funding for growth in GME to specialties and locations in short supply.

\section{Market Competition}

Some sponsoring institutions are motivated to increase their competitive edge in the health care marketplace by engaging in GME, while others are reluctant to engage in GME in markets where a similar GME program already exists. Large health systems will carefully consider competition in each local market when deciding to establish or expand a GME program in that community. Furthermore, doctors who finish training then choose to work for a competitor are sometimes viewed negatively, even if they are boosting the overall number of needed physicians in the community.

Yeah, in family medicine we need more residents, but I'm not going to pay to train residents for [competitors]. I mean, if they're not going to stay [at our institution], in reality, I would just as soon shrink the program by half. -Administrator \#1, Teaching Hospital

\section{Potential Clinical Revenue}

Whether or not residents and fellows are able to generate enough clinical revenues to offset the entire cost of their training remains a topic of much debate. Revenues vary substantially by specialty, with procedure-based specialties such as orthopedics and dermatology, generating more clinical income relative to lesser-compensated specialties such as family medicine and psychiatry. The perception of some specialties as revenue-generators and others as cost-centers can influence which programs are offered.

And so the value of the orthopedic program is that it's lucrative for the hospital. ...it's just the derivative benefit of all that hospital surgical care. - Designated Institutional Official \#2

\section{Academic Stature}

For large academic health centers (AHCs), prestige among peer institutions is important. As tertiary and quaternary referral centers, $\mathrm{AHCs}$ tend to focus on training subspecialists and fellows. Despite high demand for family medicine and psychiatry physicians in the community, an AHC might constrain the number of residency positions in order to accept only the most competitive applicants, choosing instead to invest institutional dollars in training physicians in a new field of medicine or an emerging subspecialty to improve their academic stature.

Academic stature works slightly differently for smaller, community-based sponsoring institutions. Simply advertising that they have a GME program can improve their status and reputation within the community since popular opinion is that teaching hospitals often have the latest cutting-edge technology, research, treatments, better doctors, and better outcomes.

We've gotten more and more publicity and prominence over the last few years around what we do. -Program Director

\section{Local Workforce Demand}

Meeting workforce demand, both within the institution and within the local community provides an important incentive for engagement in GME. GME programs boost recruitment and retention of high caliber faculty physicians, and produce graduates willing to stay and practice at the institution and in the local community.

Our strongest objective is to train quality physicians... for retention purposes. - Board Member, Teaching Health Center

\section{Mission and Culture}

Some institutions are highly motivated to engage in, and expand, GME because education is a core part of their mission and culture. For example, other organizations place a higher priority on patient access, patient throughput, or physician productivity.

[Our $\mathrm{FQHC}$ ] mission is patient care, output volume, expanding services. The mission of residency is 
actually not directly patient care..... [Residents'] productivity is lower; their need to be in various places besides the clinic is higher. And so the educational mission and the FQHC mission sometimes don't happily coexist. -Designated Institutional Official \#2

\section{Staffing}

Sponsoring institutions engaged in GME often depend on residents and fellows to staff their hospitals and clinics. Faculty physicians expect trainees to work long hours, including evenings, weekends, and on-call hours, and depend on trainees to handle many of the first-line patient care responsibilities, thereby offloading the work of the faculty.

As a resident, they can work... up to 80 hours a week. And their salary is not that much at all. I mean, I think we did a calculation one time. For the amount of hours they truly work and what they make, they make barely over minimum wage ... And so you can choose to have a training program and pay for all those people, or you can hire a physician, a nurse practitioner... which is going to cost your hospital a lot more. -Designated Institutional Official \#3

\section{Financial Reserves}

Starting a new GME program, or expanding an existing program beyond its Medicare cap, can be challenging, as funding is typically not covered by public subsidies. Some sponsoring institutions have substantial financial reserves that they can use to pay for start-up or expansion costs. For instance, nonprofit hospitals can expend community benefit dollars on GME. Other institutions rely almost completely on outside funding.

That's just a critical factor that is really difficult for foundations, or I think even HRSA, the management of [the residency] or project managers to understand: that gearing up time. It's $\$ 100,000$ worth of work that has to be done before you can even get started with putting together your program. -Administrator \#1, Teaching Health Center

\section{Educational Leadership}

Any new sponsoring institution needs an educational champion to help overcome the many challenges during the startup period and accreditation process. This person may or may not serve as the required designated institutional officer (DIO) who oversees and is ultimately responsible for all aspects of GME.

It's a combination of strong interest by, say, the department and maybe a few leaders who really have a passion around GME.... Sometimes it just takes a person with a lot of passion and energy to make it happen. - Administrator \#2, Teaching Hospital

Also required are program directors with sufficient training and dedicated paid time to administer each individual GME specialty program at the sponsoring institution, and faculty clinicians. Some clinicians do not want to serve as faculty because teaching can have a negative impact on personal income (from less clinical revenue) and result in longer hours. Others view teaching as a benefit that enhances job satisfaction and reduces burnout by adding diversity to their workday.

\section{Teaching Resources}

Institutions need sufficient resources to start or sustain a teaching program, including an adequate supply of patients with the appropriate variety and severity of illnesses and injuries. Some specialties require the presence of another accredited GME program. Core infrastructure must also be in place, from support personnel, such as the program coordinator, to specialty equipment, such as simulators, to dedicated space, such as resident call rooms and workstations. Sometimes this means collaborating with an outside institution, for example, a teaching health center may partner with a hospital to provide residents the required inpatient experiences.

'What does it take to get off the ground?' Money was one of them, but it was not the limiting factor in many cases. Many cases it's, 'Can you meet the ACGME requirements? Do you have the faculty? Do you have the rotations? Do you have the relationships with the hospitals?' -Administrator \#2, Teaching Health Center

Size

The size of a sponsoring institution can influence its capacity to engage in GME. Because some GME investments are the same regardless of the size of the program, and some resources can be shared across multiple specialties, sponsoring institutions can benefit-to a point-from economies of scale. Small sponsoring institutions often need to form partnerships in the community to establish new, or expand existing, programs.

Even though we're small, we still have all of the same requirements of a big program, so the same level of support, administrative staff. We have to meet all of the guidelines. - Designated Institutional Official \#4

\section{Discussion and Implications}

Sponsoring institutions play a central role in shaping the physician workforce through their decisions to engage in GME. They make these decisions in the context of their organization and community, with little regard for national or regional workforce planning. They are understandably concerned about staffing their clinical services, gaining market share, improving their reputation, and maximizing their clinical revenues. They weigh these decisions in light of limited resources including financial reserves, educational leadership, and other teaching resources. So how does this information add to our thinking about GME expansion to meet well-recognized physician 
workforce needs in terms of specialty and geographic location? It is helpful to consider which of these factors that drive GME decision-making within sponsoring institutions can best be influenced by state or federal policy change. Market competition is difficult to shape via regulatory policies. The wide variation in clinical revenues and prestige across specialties is deeply rooted; policy changes needed to affect change in those factors are beyond the scope of this study. Medicare and other GME subsidies are perhaps the most effective policy levers for GME expansion in areas of greatest need. A very small proportion of the $\$ 16$ billion in federal GME funding is tied to national physician workforce goals. The National Academy of Medicine has called for an overhaul of federal GME funding that would tie Medicare and other federal GME subsidies to national workforce goals, but the current antiquated system is set in statute, and major change has proven difficult due to lack of consensus among stakeholders. Until meaningful change occurs, strategies for GME expansion need to function within the limitations of the current Medicare rules and, where possible, incorporate measures of outcomes and accountability.

\section{Medicare GME Subsidies as a Lever for GME Expansion}

One way of expanding GME consistent with workforce goals under the current Medicare rules is to establish new GME programs in hospitals that have never had a teaching program (also called Medicare GMEnaïve hospitals) and are therefore eligible for new Medicare GME funding. For example, the State of Georgia provided start-up funding to incentivize Medicare GME-naïve hospitals to begin teaching programs in key specialties. By 2025 this state program is projected to have resulted in the opening of 27 programs in eight new teaching hospitals with 613 new residency positions. As part of the funding guidelines, the state was able to stipulate the types of residency programs allowed, resulting in new programs in specialties of physician shortages. ${ }^{10-13}$ For sponsoring institutions in Georgia, the influx of new Medicare GME subsidies served as a strong incentive to launch GME programs.

\section{Medicaid GME Subsidies as a Lever for GME Expansion}

Many states are experimenting with using Medicaid GME subsidies as a lever for workforce planning. New Mexico provides one innovative example. Through their Medicaid program, New Mexico successfully financed the New Mexico Primary Care Training Consortium in 2013, subsequently approving and reallocating funding for primary care GME. One key aspect of the program was that it allowed Medicaid payments to provide for residency training at FQHCs, including the development of new residency programs and expansion of existing ones. ${ }^{14}$

Although most states recognize that explicit Medicaid GME subsidies with increased accountability can be an important policy lever to advance specific workforce goals, progress in this area varies substantially between states; because each state Medicaid GME program has its own history and politics, it can be difficult to replicate or model innovations from one state to another.

\section{Veterans Affairs (VA) Funding as} a Lever for GME Expansion

Although the VA has radically reduced its role as a sponsoring institution, VA medical centers have access to considerable funds to implement GME in partnership with other sponsoring institutions. The workforce goals of the VA typically overlap with those of the United States, emphasizing, for example, primary care, geriatrics, and psychiatry.

\section{State Subsidies as a Lever for GME Expansion}

States can prioritize GME that aligns with workforce goals through state funding sources, such as tobacco taxes, insurer and/or provider assessments, and general funds. These funds can sometimes be used to draw down additional federal dollars through the Medicaid program, or they can be used to fund GME programs directly. As an example, Texas has made a large investment in expanding its GME programs, appropriating roughly $\$ 183$ million in fiscal year 2018-2019 from its general fund (and another $\$ 21.8$ million from its endowment fund, the GME Permanent Fund) to support residencies and GME expansion programs. Through state involvement, Texas has reached its goal of a 1.1 to 1 ratio of first-year residency positions to medical school graduates, successfully promoting growth in underrepresented specialties and geographic areas. Similarly, California directly funds its Song-Brown Program to support primary care GME, particularly in underserved areas, and has recently begun to use tobacco tax funds to expand primary care and emergency medicine GME.

\section{Accountability}

Sponsoring institutions understandably make GME decisions with little consideration of national or regional workforce needs, but rather in alignment with their organizational priorities. If public funds are used as a policy lever to expand GME in shortage specialties and locations, accountability is key. Outcomes metrics should not be limited to fiscal accountability and quality of training, but should also include workforce outcomes such as physician race, ethnicity, language, specialty, and location of practice. Unless and until the incentives for sponsoring institutions are strongly aligned with national and state physician workforce priorities based on public need, the prerogatives of individual sponsoring institutions will continue to eclipse public planning in shaping the nation's physician workforce. 


\section{*Footnote:}

A sponsoring institution is an organization that assumes the ultimate financial and academic responsibility for GME. Sponsoring institutions may include teaching hospitals, schools of medicine, Federally Qualified Health Centers (FQHCs), and other types of organizations accredited by the Accreditation Council for Graduate Medical Education (ACGME). There were approximately 830 sponsoring institutions in the United States in 2018.

FUNDING STATEMENT: This article was published with support from the California Health Care Foundation. More information available at https://www.chcf.org/.

CORRESPONDING AUTHOR: Address correspondence to Dr Diane R. Rittenhouse, Mathematica, 505 14th Street, 8th Floor, Oakland, CA 94612. 510-285-4620. DRittenhouse@mathematica-mpr.com

\section{References}

1. Congressional Research Service. Federal Support for Graduate Medical Education: An Overview. Washington, DC: Congressional Research Service; updated December 27, 2018. https://fas.org/sgp/crs/misc/R44376.pdf. Accessed July 9, 2020

2. Salsberg E, Armstrong A, Forte G. Demand for Family Medicine Physicians Ranks Highest Among Specialties. Health Affairs Blog. August 18, 2016. https://www.healthaffairs. org/do/10.1377/hblog20160818.056187/full/. Accessed July 9, 2020
3. Grover A, Orlowski JM, Erikson CE. The Nation's Physician Workforce and Future Challenges. Am J Med Sci. 2016;351(1):1119.

4. Association of American Medical Colleges. 2019 Update: The Complexities of Physician Supply and Demand: Projections from 2017 to 2032. Washington, DC: AAMC; Apri 2019. https://aamc-black.global.ssl.fastly.net/ production/media/filer_public/31/13/3113ee5ca038-4c16-89af-294a69826650/2019_update_-_the_complexities_of_physician_supply_and_demand_-_projections_from_20172032.pdf. Accessed July 9, 2020

5. Khatib D, Huffstetler A, Bazemore A. Addressing Rural Workforce Shortages and Healthcare Disparities: An Annotated Bibliography. Washington, DC: Robert Graham Center; February 2019. https://www.grahamcenter.org/content/dam/rgc/documents/publications-reports/reports/RuralHealth-AnnotatedBibliography-COGME.pdf. Accessed July 9, 2020.

6. Robert Graham Center. The State of Primary Care Physician Workforce. Washington, DC: Robert Graham Center; January 2019. https://www.graham-center.org/content/dam/ rgc/documents/publications-reports/reports/ StateFactSheetReport.pdf. Accessed July 9, 2020 .

7. Committee on the Governance and Financing of Graduate Medical Education; Board on Health Care Services; Institute of Medicine; Eden J, Berwick D, Wilensky G, eds. Graduate Medical Education That Meets the Nation's Health Needs. Washington, DC: National Academies Press, September 30, 2014. https://www.ncbi.nlm.nih.gov/books/ NBK248027/. Accessed July 9, 2020.

8. Mullan F, Chen C, Steinmetz E. The geography of graduate medical education: imbalances signal need for new distribution policies. Health Aff (Millwood). 2013;32(11):1914 1921.
9. Nuss MA, Robinson B, Buckley PF. A Statewide Strategy for Expanding Graduate Medical Education by Establishing New Teaching Hospitals and Residency Programs. Acad Med. 2015;90(9):1264-1268.

10. Kornegay D. GME in Georgia: Growth, Funding, and Sustainability. Augusta, GA: Augusta University; October 18, 2016. https://www.augusta.edu/ahec/documents/ gme_white_paper.pdf. Accessed July 9, 2020.

11. Nuss S. GME Regents Evaluation and Assessment Team (GREAT): Guiding Principles. February 24, 2014.

12. Kaufman A, Alfero C. A State-Based Strategy for Expanding Primary Care Residency. Health Affairs: July 2015. https://www.healthaffairs.org/do/10.1377/ hblog20150731.049707/full/. Accessed July 9, 2020. 